\title{
Copper decreases associative learning and memory in Drosophila melanogaster
}

\author{
D. C. Zamberlan; P. T. Halmenschelager; L. F. O. Silva; J. B. T. da Rocha
}

\begin{abstract}
Copper is an essential element to all living organisms. Repeated use of metal-enriched chemicals, fertilizers, and organic substances may cause contamination at a large scale. Altered levels of $\mathrm{Cu} 2+$ may result in harmful effects and can be associated with memory and cognitive dysfunction. Studying simple, genetically tractable organisms such as Drosophila melanogaster, can reveal important data on the neural basis of conditioning. D. melanogaster is an important alternative experimental model to assess the toxic response to metals. In the present study, the effects of copper on flies' development and in learning and memory retention in male and female adult flies were investigated. We paired an odorant to pain perception and observed the aversion behavior over time. Exposure of D. melanogaster eggs to Cu2+ increased mortality of larvae, pupae, and adults and decreased memory retention in adults. Moreover, male flies demonstrated to be more susceptible to Cu2+ toxicity than females. The results therefore, reinforce the importance of controlling the anthropogenic heavy-metals soil contamination given their hazardous effects to living organisms.
\end{abstract}

Keywords: invertebrates, metal contamination, classical conditioning, toxicity, flies development. 